\title{
Excess mortality and cardiovascular disease in type 1 diabetes in relation to age at onset: a nationwide study of 27,195 young adults with diabetes
}

\author{
Araz Rawshani ${ }^{1,{ }^{*}}$, Professor Naveed Sattar ${ }^{2,{ }^{*}}$, Stefan Franzén ${ }^{3}$, Aidin Rawshani ${ }^{4}$, Professor \\ Andrew T Hattersley, Ann-Marie Svensson ${ }^{3}$, Professor Björn Eliasson ${ }^{5}$, Professor Soffia \\ Gudbjörnsdottir ${ }^{2,3}$ \\ ${ }^{1}$ Department of Molecular and Clinical Medicine, Institute of Medicine, University of Gothenburg, \\ Gothenburg, Sweden \\ 2Institute of Cardiovascular and Medical Sciences, University of Glasgow, UK \\ ${ }^{3}$ The Swedish National Diabetes Register, Västra Götalandsregionen, Gothenburg, Sweden \\ ${ }^{4}$ The Sahlgrenska University Hospital, Gothenburg, Sweden; Institute of Biomedical \& Clinical \\ Science, University of Exeter Medical School, Exeter, UK \\ ${ }^{5}$ Department of Internal Medicine, Institute of Medicine, University of Gothenburg, Gothenburg, \\ Sweden
}

\section{Abstract}

Background-We compared individuals with type 1 diabetes (T1D) to matched controls in order to examine how age at diagnosis of T1D relates to excess mortality and cardiovascular (CV) risk.

Methods-We studied 27,195 persons with T1D in the Swedish National Diabetes Registry, and 135,178 matched controls from the general population. Using Cox regression, and with adjustment for diabetes duration, we estimated excess risk of all-cause mortality, $\mathrm{CV}$ mortality, non-CV mortality, acute myocardial infarction (AMI), stroke, CVD (AMI and stroke), coronary heart disease (CHD), heart failure (HF) and atrial fibrillation (AF). Individuals with T1D were categorized into five groups, according to age at diagnosis: $0-9,10-14,15-19,20-24$ and 25-30 years.

\footnotetext{
*Corresponding authors: Araz.rawshani@gu.se, Naveed.Sattar@glasgow.ac.uk.

* Joint first authors

Contributors

Ar.R, N.S, Ai.R and SG contributed to study concept and design. S.F performed the statistical analyses. Ar.R, N.S had the primary responsibility for writing the paper. ATH added new data interpretation. All authors reviewed and revised subsequent versions of the manuscript. Ar.R, N.S, S.F, Ai.R and S.G vouch for the integrity of the analyses.

Declaration of interests

NS has consulted for Boehringer Ingelheim, Novo Nordisk, Janssen and Eli Lilly, and received grant support from AstraZeneca. BE has received personal fees (advisory panels and/or consultant) from Amgen, AstraZeneca, Boerhringer Ingelheim, Eli Lilly, Merck Sharp \& Dohme, Mundipharma, Navamedic, Novo Nordisk, and RLS Global outside the submitted work, and grants from Sanofi outside the submitted work. SG has received personal fees (lecture fees and research grants) from AstraZeneca, Boerhringer Ingelheim, Eli Lilly, Merck Sharp \& Dohme, Novo Nordisk, Sanofi outside the submitted work. Ar.R has received personal fees from Novo Nordisk.
} 
Findings-A total of 27,195 persons with T1D and 135,178 controls were included; 924 persons with T1D and 1,405 controls died during follow-up, of which median was 10 years. Patients who developed T1D at 0-10 years of age displayed hazard ratios (95\% CI) of 4.11 (3.24-5.22) for death, 7.38 (3.65-14.94) for CV death, 11.44 (7.95-16.44) for CVD, 30.50 (19.98-46.57) for CHD, 30.95 (17.59-54.45) for AMI, 6.45 (4.04-10.31) for stroke, 12.90 (7.30-22.51) for HF and $1.17(0.62-2.20)$ for AF. Corresponding figures for those who developed T1D in the age-range 2630 were 2.83 (2.38-3.37) for death, 3.64 (2.34-5.66) for CV death, 3.85 (3.05-4.87) for CVD, 6.08 (4.71-7.84) for CHD, 5.77 (4.08-8.16) for AMI, 3.22 (2.35-4.42) for stroke and 5.07 (3.557.22) for HF; hence excess risk differed up to 5-fold across the diagnosis age. The highest overall incidence rate, noted for all-cause mortality, was 1.9 (95\% CI 1.71 to 2.11) per 100.000 personyears for patients with T1D. Developing T1D before 10 years of age resulted in a loss of 17.7 and 14.2 life years for women and men, respectively, whereas years lost were 10.1 and 9.4 in those diagnosed between 26-30 years of age.

Interpretation-Age at onset of type 1 diabetes is an important determinant of survival, as well as all cardiovascular outcomes, with highest excess risk in females. Greater focus on cardioprotection maybe warranted in those with early onset T1D.

Funding_-Swedish Heart and Lung Foundation

\section{Introduction}

Type 1 diabetes is the second most common chronic disease of childhood, although the disorder may develop throughout the life span. ${ }^{1}$ Remarkable improvements in management and survival has been observed during the past century. A recent study demonstrated that the relative risk of death declined by $29 \%$ over a 10 -year period. Yet, mortality in type 1 diabetes is still increased two- to eightfold, ${ }^{2,3}$ which is reflected by a loss of life expectancy at age 20 years of approximately 12 years. ${ }^{4}$ Cardiovascular disease is the main driver of morbidity and mortality in people with type 1 diabetes. Guidelines therefore recommend aggressive management of cardiovascular risk factors in type 1 diabetes, especially once beyond 40 years of age or with evidence of microvascular complications. ${ }^{5}$ Yet, guidelines are not well adhered to in type 1 diabetes and even with risk factors at target, people with type 1 diabetes are at elevated risk of mortality and cardiovascular disease. ${ }^{6}$ No current guideline considers age of onset as an important risk stratifier.

Age at diagnosis may be important in type 1 diabetes. It may carry information on-and thus act as a proxy for-several important factors, such as total glycemic load, varying autoimmune mechanisms, age-related variations in clinical care, differences in ability to cope with the disease etc. Accordingly, recent studies have demonstrated that age at diagnosis can contribute to identifying subtypes of type 2 diabetes in adults, ${ }^{7}$ as well as predict risk factor trajectories. ${ }^{8}$ Furthermore, other evidence supports younger onset type 2 diabetes being more harmful than diabetes diagnosed in later life. 8,9

By contrast, such data in type 1 diabetes are less evident. ${ }^{10-14}$ No study has examined how age at diagnosis relates to excess risk of death and cardiovascular outcomes, while accounting for duration of diabetes, and using such granular age categories. We studied 27,195 individuals with T1D and 135,178 matched controls to answer this research question. 


\section{Methods}

\section{Data sources and study population}

The Swedish National Diabetes Register (NDR), ${ }^{2,6,15}$ includes longitudinal data regarding risk factors, complications, treatment and management for virtually all individuals with type 1 diabetes aged 18 years and older. Virtually all Swedes $\geq 18$ years of age with type 1 diabetes are enrolled in the registry. Type 1 diabetes is defined for the NDR on the basis of epidemiological data: treatment only with insulin and a diagnosis at $₫ 30$ years of age, which has been validated as accurate in $97 \%$ of cases. Validation was done by comparing the concordance between the epidemiological classification and the physician's classification of diabetes type. ${ }^{16,17}$ We included patients with at least 1 registration between January 1, 1998, and December 31, 2012. For the baseline (i.e the first registration in the NDR) each patient was matched for age, sex, and county with 5 controls (without diabetes mellitus) randomly selected from the Swedish population, as previously done. ${ }^{2,15,18}$ Matching on county aimed at reducing geographical differences in characteristics.

We excluded patients with type 1 diabetes, along with their controls, if the former had severe congenital disorders or syndromes that may bias the association between diabetes and outcomes. A complete list of these disorders is provided at the end of the supplementary appendix. If any individual in the matched set (consisting of 1 individual with type 1 diabetes and 5 matched controls) had such conditions, the entire matched set was excluded. This led to exclusion of $0.6 \%$ of the originally eligible individuals. We also excluded individuals with inconsistent vital data (i.e the registration in the NDR was dated after time of death), which resulted in exclusion of 255 patients with type 1 diabetes, along with their controls. Finally, we excluded individuals with more than 20 years duration of diabetes, the reason for which is explained below. Finally, 27,195 persons with type 1 diabetes and 135,178 matched controls were studied. Supplementary Tables 1 and 2 presents the number of individuals excluded due to congenital disorders and inconsistent vital data.

\section{Covariates, coexisting conditions and causes of death}

Information on socioeconomic data, coexisting conditions, dates and causes of death was retrieved by linking data to Statistics Sweden, the Swedish Inpatient Registry and the Cause of Death Register, respectively. Data linkage is seamless since all Swedish citizens are assigned to a personal identification number which is used in these registries.

Statistics Sweden includes information regarding annual income, country of birth, marital status and education. The Inpatient Register includes all hospital admissions since 1987. Primary and secondary discharge diagnoses are coded according to the International Classification of Diseases (ICD). We assessed ICD-9 and ICD-10 codes to define the following coexisting conditions: coronary heart disease: 410-414 (ICD-9), I20-I25 (ICD-10); acute myocardial infarction: 410 (ICD-9), I21 (ICD-10); stroke: 431-434, 436 (ICD-9), I61I64 (ICD-10); hospitalization for heart failure: 428 (ICD-9), I50 (ICD-10); atrial fibrillation: 427D (ICD-9), I48 (ICD-10); cancer: 140-208 (ID-9), C00-C97 (ICD-10). For each outcome, only the first recorded event in the Inpatient Register was assessed. The validity and reliability of these diagnoses in the Inpatient Register has been examined in detail. ${ }^{19}$ 


\section{Outcomes and exposures}

We estimated the excess risk of all-cause mortality, cardiovascular (CV) mortality, noncardiovascular mortality, acute myocardial infarction (AMI), stroke, CVD (composite of AMI and stroke), coronary heart disease (CHD), heart failure (HF) and atrial fibrillation (AFib). Individuals with type 1 diabetes were categorized into five groups, according to age at diagnosis: 0 to 10 years, 11 to 15 years, 16 to 20 years, 21 to 25 years and 26 to 30 years.

For each matched set (the patient with T1D and the matched controls), follow-up started on the date of the patient's first registration in the NDR, and ended on date of event, death, emigration or end of follow-up (2014-12-31).

\section{Statistical methods}

Cox regression was used to study the association between age at diagnosis of diabetes and risk of the outcomes, compared with controls. Age was used as the underlying time-scale, allowing the baseline hazard to capture the increase in hazard due to aging. For individuals with type 1 diabetes, we centralized duration around its grand mean. Controls were modelled as individuals not yet diagnosed with diabetes, meaning that their duration of diabetes was set to zero; this allows us to account for duration without assigning an effect of duration to controls. The resulting hazard ratios represent the hazard ratio for each group after the average duration of diabetes, which was 13 years. In order to compute reliable estimates, we had to exclude patients with duration above 20 years. Limiting duration to 20 years allowed for computation of simple models, without the need for relaxing assumptions of linearity in duration and it also yielded the best overlap in distribution of duration between the diabetes groups. Moreover, limiting duration to 20 years also allows us to study a more contemporary cohort, which better reflects modern diabetes management.

In all models, we adjusted for age (using it as time scale), sex, marital status, income, educational level, region of birth, duration of diabetes, previous histories of AMI, stroke, CVD, CHD, atrial fibrillation and heart failure.

This was done in the entire cohort (i.e including people with $>20$ years duration of diabetes). The life years lost was estimated as the difference between the predicted conditional median survival post 18 years of age in the 0-10 years and 10-15 years group and post the upper age interval for the other groups. The predicted conditional survival functions were derived from a Cox regression model with T1DM vs controls as the only independent variable. Age was used as the time scale, with left censoring at age of inclusion. The conditional median survival was estimated from the upper limit of each age interval.

The ethics committee of the University of Gothenburg, Sweden, approved the study. All patients with diabetes have provided informed consent before inclusion in the cohort. The funder had no role in any part of the study.

\section{Results}

A total of 27,195 persons with type 1 diabetes and 135,178 controls were included. Median follow-up was 10 years; 924 patients with type 1 diabetes and 1,405 controls died during 
follow-up. Follow-up time and number of deaths in each age category is presented in Supplementary Tables 3 and 4

\section{Baseline characteristics}

Patients vs. controls-Mean age among patients with T1D and controls was roughly 29 years and $56 \%$ were males (Table 1). There were only small differences in educational attainment and marital status between controls and patients. Controls earned 4700 SEK more per year (approximately 550 USD or 405 GBP). All coexisting conditions, with the exception of atrial fibrillation, were more common in patients with diabetes. Coronary artery disease at baseline was 9 times as common in patients with type 1 diabetes.

Patients with diabetes-Mean ages in the five age-groups were 23.5, 25.7, 27.6, 32.2 and 37.9 years (Table 2). HbA1c values were higher in patients with younger age at onset. Use of antihypertensives and statins were lowest in those with low age at onset of type 1 diabetes. Blood pressure, triglycerides, BMI, LDL cholesterol and prevalence of smoking increased with age at diagnosis. There were only small differences in physical activity and prevalence of micro- and macroalbuminuria.

\section{Absolute risk estimates}

Supplementary Tables 5 and 6 present incidence rates for all outcomes. Incidence rates were low, mostly below 2 events per 100.000 person-years. Rates increased with age-group. The highest overall incidence rate was noted for all-cause mortality, being 1.9 (95\% CI 1.71 to 2.11) per 100.000 person-years for patients with type 1 diabetes and 0.6 (95\% CI 0.56 to 0.66) for corresponding controls. In the highest age-group, incidence rates for CVD and CHD were 1.53 (95\% CI 1.35 to 1.73 ) and 1.8 (95\% CI 1.61 to 2.01 ) per 100.000 personyears, respectively, for patients, and 0.45 (95\% CI 0.41 to 0.5 ) 0.46 (95\% CI 0.41 to 0.5 ) for controls. Corresponding rates for those who developed diabetes below 10 years of age were $0.48(0.38,0.58)$ and $0.5(0.41,0.61)$ per 100.000 person-years.

\section{Life years lost}

There were marked differences in life years lost. Refer to Figure 1 and Supplementary Tables 7 through 9. Overall, being diagnosed with type 1 diabetes before 10 years of age resulted in loss of 16.0 (95\% CI 15.7, 16.4) life-years. Men who were diagnosed before 10 years of age lost 14.2 (95\% CI 14.4, 15.8) life-years. Females who were diagnosed at the same age lost 17.7 (95\% CI 17.1, 17.8) life-years. Patients diagnosed after 20 years of age loss approximately 10 life-years.

\section{Hazard ratios for mortality and cardiovascular outcomes}

All following hazard ratios represent the risk in people with type 1 diabetes, according to age at diagnosis, compared with controls. Overall hazard ratios refer to estimates for men and women collectively. Sex-specific hazard ratios are also provided.

General patterns-Patients with diabetes displayed an excess risk of eight of nine outcomes, with atrial fibrillation being the only exception. There was a ubiquitous inverse association between age at diagnosis and risk of the outcomes. Excess risks were 
particularly pronounced in women; the greatest risks were noted for coronary artery disease and myocardial infarction, for which women with type 1 diabetes displayed a 60- and 90fold increased risk, respectively.

Mortality-Figure 2 presents overall hazard ratios (men and women collectively). Hazard ratios (95\% CI) for all-cause mortality were as follows: 4.11 (3.24 - 5.22), 3.21 (2.58 - 4.00), 3.02 (2.44 - 3.73), 2.90 (2.41 - 3.50) and 2.83 (2.38 - 3.37), going from the youngest age-group to the oldest. CV mortality displayed a similar trend. The lowest hazard ratio for CV mortality, noted for those with diabetes onset at age 26-30 years, was 3.64 (2.34 - 5.66). The highest hazard ratio was $7.38(3.65-14.94)$ and noted for those with disease onset at age $0-10$ years. Excess risk of non-CV mortality was consistently elevated with an incremental increase (albeit less marked than for CVD) with youngers age at onset of T1D. Hazard ratio for patients diagnosed in the age range 26-30 years was 2.78 (2.293.38). For those diagnosed in the age-range $0-10$ years the hazard ratio was 3.96 (3.065.11).

Figure 3 presents hazard ratios according to sex. There were no material differences between males and females (with type 1 diabetes) in the age-range 20 to 30 years. However, women displayed greater hazard ratios when developing diabetes before 20 years of age. For those developing type 1 diabetes before 10 years of age, women displayed a 6-fold increased mortality risk, as compared with a 3 -fold risk noted in males with type 1 diabetes.

Cardiovascular outcomes-Cardiovascular risks were considerably higher and strongly related to age at disease onset. Several strong associations were noted.

Overall hazard ratio for CVD (Figure 2) for patients diagnosed in the age-range 26-30 years was 3.85 (3.05-4.87), whereas patients diagnosed in the age-range $0-10$ years displayed a hazard ratio of 11.44 (7.95-16.44). There were notable differences between males and females, such that the latter displayed greater excess risk throughout (Figure 3). Women who developed type 1 diabetes before 10 years of age had a 13-fold increased risk of CVD.

Overall hazard ratio for CHD was 30.50 (19.98-46.57) for those diagnosed in the age-range $0-10$ years (Figure 2). The lowest hazard ratio for CHD was 6.08 (4.71-7.84), which was noted for those diagnosed in the age-range 26-30 years. There were differences between males and females with type 1 diabetes (Figure 3 ). Males with type 1 diabetes displayed a hazard ratio of 16.95 (10.03-28.67) and the corresponding figure for females was 58.73 (28.86-119.55).

Overall hazard ratio for AMI was 30.95 (17.59-54.45) for those diagnosed in the age-range 0-10 years (Figure 2). Women who developed type 1 diabetes before 10 years of age had a hazard ratio of 91.07 (32.72-253.47). The corresponding figure in males was 15.11 (7.5330.33). These differences persisted further down the age span (Figure 3). The lowest hazard ratio for women with type 1 diabetes was 14.13 (7.75-25.76), which was noted for those with disease onset between age $26-30$ years.

Overall hazard ratio for stroke was $6.45(4.04-10.31)$ for disease onset between $0-10$ years of age (Figure 2). This excess risk declined gradually with increasing age at onset, such that 
those with disease onset between 26-30 years had a hazard ratio of 3.22 (2.35-4.00). There were no significant differences between men and women with type 1 diabetes (Figure 3).

Hazard ratios for heart failure differed by a factor of two across the age-span. The lowest hazard ratio was 5.07 (3.55-7.22) and noted for the age-group 26-30 years (Figure 2). Being diagnosed with type 1 diabetes in the age-range $0-10$ years resulted in a hazard ratio of 12.90 (7.39-22.51). There were no material differences between men and women with type 1 diabetes (Figure 3).

With regards to atrial fibrillation, we did not note any excess risk for patients with diabetes, except from males with disease onset between age 21-25 years.

\section{Causes of death in relation to age at diagnosis}

Circulatory and endocrine causes represented roughly $70 \%$ of all primary causes of death in those with disease onset at age $0-10$ years (Figure 4). The corresponding figure for those with age 26-30 was $61 \%$. Other causes, especially neoplasms, became more common with later onset T1D.

\section{Discussion}

In this nationwide study of patients with type 1 diabetes we show that age at disease onset is an important determinant of survival and cardiovascular disease. The differences in hazard related to age of disease onset were in many cases extreme. Patients with type 1 diabetes with disease onset before 10 years of age experienced a 30-fold increased risk of CHD and AMI in their early adult years. Women with onset of type 1 diabetes before 10 years of age displayed a 60- and 90-fold increased risk of CHD and AMI, respectively, in the same early adult period. Onset of type 1 diabetes before 10 years of age was associated with 12-fold increased risk of heart failure over the same period and mortality risks, relative to age and sex matched controls, differed by $128 \%$. It is important to note that although the relative risks were extremely high, absolute risks were low throughout. This is explained by the fact that we studied a relatively young cohort (mean age 29). However, our previous studies, in which the persons with T1D were approximately 8 years older than in the present study, we demonstrated that absolute risks were much higher. ${ }^{3}$ Hence, if the relation between age at diagnosis and excess risks persist further into the future, it would in time translate to a very high absolute risk, as corroborated by our analysis of loss of life years; women who developed type 1 diabetes before 10 years of age lost almost 18 life-years. A diagnosis at 26-30 was associated with around 10 years loss.

In the light of the fact that around half of type 1 diabetes cases are diagnosed before 14 years of age, ${ }^{20}$ this study highlights a need to consider age at diagnosis in guidelines. The magnitude of these risk estimates - with point estimates approaching 100 in the early adult years - appear at least as high as those conferred by familial hypercholesterolemia. ${ }^{21}$

Our risk estimates for the 0-10 years of age of onset subgroup are higher than figures presented in the most recent statement from the American Heart Association and American Diabetes Association, which noted that patients with T1D were at 3- (men) and 7-fold 
(women) increased risk of CHD. Notably, this statement did not consider age of onset as a risk stratifier, whereas our data suggest age of onset should now be considered in the management of CVD risk in T1DM.

Our data suggests that excess risks are generally greater in women developing T1D (with the exception of heart failure). This can, to some extent, be explained by the fact that women in the general population have a low risk of these events, as compared with men. Hence, higher excess risks in women with type 1 diabetes do not necessarily translate into higher absolute risks compared to their male counterparts. ${ }^{22}$

Our data add meaningfully to the limited information available on the long-term survival in type 1 diabetes in relation to age at disease onset. Conway and colleagues compared mortality in diabetes with onset in childhood (under 20 years of age, $n=162$ ) versus young adulthood (20 to $29, \mathrm{n}=313$ ); no marked differences in mortality or coronary artery disease were noted, although the small sample offered low power. ${ }^{14}$ In a bigger study, Harjutsalo and colleagues examined CHD mortality in early (0-14 years) and late onset (15-29) T1D. They reported that CHD mortality rate was 2.8 -fold greater in early onset type 1 diabetes compared with late onset type 1 diabetes. Harjutsalo et al suggested that this higher risk may be explained by the longer duration of diabetes in those with early onset type 1 diabetes. Our study, which had the benefits of individual's controls, adjustment for duration, more age subgroups, as well as a range of CVD outcomes, demonstrates that mortality in T1D is uniformly and markedly elevated as compared with the general population.

Although the explanations for our findings are likely to be multifaceted, diabetes duration is likely to play a key role, since even though we adjusted for duration more robustly than other studies, complete adjustment is near impossible. Duration of diabetes is a component of total glycemic load. The latter-defined as the vasculatures cumulative exposure to glucose-is a function of duration of diabetes and glycemic variability. The longer the duration of diabetes, the greater the glycemic load and thus the damages (analogous to area under the curve for exposure to LDL cholesterol). ${ }^{23,24}$ It is also clear from our data that the coronary arteries seem especially vulnerable to hyperglycemia, and more so when hyperglycaemia commences early in life (under 10-15 years).

One possible recent explanation for our findings is that patients with a younger age of onset have a more severe and rapid loss of beta-cells which contributes to higher glycaemia, as we noted. Recent studies of the pancreas of patients who die close to diagnosis of diabetes show that those diagnosed under 7 years have very severe loss of residual insulin-containing islets (ICIs) compared to those diagnosed over the age of 13 years who retain $\sim 40 \%$ ICIs. ${ }^{25,26} \mathrm{~A}$ different type of insulitis is seen in these 2 subgroups: in those diagnosed under 7 there is a high proportion of CD20 B lymphocytes (CD20Hi), in contrast to those diagnosed over 13 years who have a low proportion (CD20Lo). ${ }^{25}$ This implies that the two forms of insulitis are differentially aggressive and that the patients diagnosed under 7 years with a $\mathrm{CD} 20 \mathrm{Hi}$ profile lose their $\beta$-cells at a more rapid rate. 
Moreover, children and adolescents with type 1 diabetes exhibit subclinical CVD abnormalities already after 10 years diabetes duration. This has been demonstrated using numerous methodologies. ${ }^{27-31}$

Our data suggest a need to better target cardiovascular risk in those with childhood onset type 1 diabetes. There are readily available and effective means to mitigate the risk of coronary events, notably statins, blood pressure lowering, insulin pump, continuous glucose measurement etc. Trial data suggest CVD reductions in type 1 diabetes with statins are near identical in magnitude to those with type 2 diabetes, ${ }^{32}$ whereas observational data suggest statin use markedly lower CVD risk in type 1 diabetes. ${ }^{33-35}$ More recent trials evidence from adDIT trial support an effect of ACE inhibition to lessen microalbuminuria and statins to lower lipids when prescribed in adolescence, and to do so without any short term harm. ${ }^{36}$

We are not advocating giving children with type 1 diabetes statins or ACE inhibitors but our data in conjunction with prior observations might argue for greater consideration of statins once individuals with early onset type 1 diabetes reach 30 to 40 years of age. From current data, plus prior work in Scotland, ${ }^{37}$ only around $10-20 \%$ of individuals with type 1 diabetes appear to be on statin by 40 years of age, and more than half have SBP>120 $\mathrm{mmHg}$. Some guidelines include long duration of diabetes (i.e. $>20$ years) as a reason to consider statins in 30-40 year old patients with type 1 diabetes. ${ }^{38}$ However, that such patients - who would have been diagnosed when under 10 or 20 years of age - also have highest risks of AMI and lose most life years from their diabetes, is strongly advanced by our present findings. We believe physicians might need to reconsider more complete targeting of cholesterol, blood pressure and glycemia in their younger onset patients when they reach 30-40 years of age, if possible and as clinically indicated. We appreciate ACE inhibitors are teratogenic whereas, although yet no clear data for such risks, statins are also not recommended for women planning pregnancy. Thus, some caution in women with type 1 diabetes is needed but even so, better blood pressure control without ACE inhibitors in women, improved glycaemia control and smoking cessation in such groups could meaningfully extend life expectancy in those with younger onset diabetes. Such interventions given earlier in life, increase life expectancy most, ${ }^{38}$ and some interventions (e.g. statins) have legacy effects. ${ }^{39}$ In other words, whilst short term risks are low to modest, given young age of diabetes onset, lifetime risks will be high and thus gains from preventative therapies will be greatest when given earlier in life.

There are a number of limitations to the current study. The investigation is a register study, which has limitations related to such design and data. We lacked information on glycaemic control prior to enrolment in the registry. We used an epidemiological definition of type 1 diabetes, which implies that misclassification of diabetes type is possible. However, a validation study has demonstrated that the epidemiological classification is highly reliable. ${ }^{16}$ Furthermore, we excluded patients with diabetes duration above 20 years in order to compute reliable regression models; this slightly restricts the permitted inferences but it does not affect the reliability of the estimates. Neither did this affect calculation of life years lost, since those analyses included all patients (no restriction with regards to diabetes duration; i.e virtually all Swedes with type 1 diabetes). 
To conclude, independent of diabetes duration, age at onset of type 1 diabetes appears an important determinant of survival, as well as all cardiovascular outcomes. Early onset type 1 diabetes is associated with up to 30 times increased risk of serious cardiovascular outcomes, with risk levels being 90 times higher for women with early onset diabetes, who also die around 18 years earlier than their diabetes free counterparts. These findings advance the arguments for wider and earlier use of cardioprotective agents.

\section{Supplementary Material}

Refer to Web version on PubMed Central for supplementary material.

\section{References}

1. Gale EAM. The rise of childhood type 1 diabetes in the 20th century. Diabetes. 2002; 51(12):335361. [PubMed: 12453886]

2. Rawshani A, Rawshani A, Franzén S, et al. Mortality and Cardiovascular Disease in Type 1 and Type 2 Diabetes. The New England journal of medicine. 2017; 376(15):1407-18. [PubMed: 28402770]

3. Lind M, Svensson A-M, Kosiborod M, et al. Glycemic Control and Excess Mortality in Type 1 Diabetes. The New England journal of medicine. 2014; 371(21):1972-82. [PubMed: 25409370]

4. Livingstone SJ, Levin D, Looker HC, et al. Estimated life expectancy in a Scottish cohort with type 1 diabetes, 2008-2010. JAMA. 2015; 313(1):37-44. [PubMed: 25562264]

5. de Ferranti SD, de Boer IH, Fonseca V, et al. Type 1 diabetes mellitus and cardiovascular disease: a scientific statement from the American Heart Association and American Diabetes Association. Diabetes Care. 2014; 37(10):2843-63. [PubMed: 25114297]

6. Rawshani A, Rawshani A, Franzén S, et al. Range of Risk Factor Levels: Control, Mortality, and Cardiovascular Outcomes in Type 1 Diabetes Mellitus. Circulation. 2017; 135(16):1522-31. [PubMed: 28416524]

7. Ahlqvist E, Storm $P$, Käräjämäki A, et al. Novel subgroups of adult-onset diabetes and their association with outcomes: a data-driven cluster analysis of six variables. Lancet Diabetes Endocrinol. 2018

8. Steinarsson AO, Rawshani A, Gudbjörnsdottir S, Franzén S, Svensson A-M, Sattar N. Short-term progression of cardiometabolic risk factors in relation to age at type 2 diabetes diagnosis: a longitudinal observational study of 100,606 individuals from the Swedish National Diabetes Register. Diabetologia. 2018; 61(3):599-606. [PubMed: 29318343]

9. Huo L, Magliano DJ, Rancière F, et al. Impact of age at diagnosis and duration of type 2 diabetes on mortality in Australia 1997-2011. Diabetologia. 2018; 61(5):1055-63. [PubMed: 29473119]

10. Dahlquist G, Källén B. Mortality in childhood-onset type 1 diabetes: a population-based study. Diabetes Care. 2005; 28(10):2384-7. [PubMed: 16186267]

11. Diabetes Control and Complications Trial (DCCT)/Epidemiology of Diabetes Interventions and Complications (EDIC) Study Research Group. Mortality in Type 1 Diabetes in the DCCT/EDIC Versus the General Population. Diabetes Care. 2016; 39(8):1378-83. [PubMed: 27411699]

12. Morgan E, Black CR, Abid N, Cardwell CR, McCance DR, Patterson CC. Mortality in type 1 diabetes diagnosed in childhood in Northern Ireland during 1989-2012: A population-based cohort study. Pediatr Diabetes. 2018; 19(1):166-70. [PubMed: 28548453]

13. Harjutsalo V, Maric-Bilkan C, Forsblom C, Groop P-H. Impact of sex and age at onset of diabetes on mortality from ischemic heart disease in patients with type 1 diabetes. Diabetes Care. 2014; 37(1):144-8. [PubMed: 24062319]

14. Conway BN, Lopes-Virella MF, Blot WJ. Late adulthood mortality among African-American and white American people with Type 1 diabetes according to age at diabetes diagnosis. Diabetic medicine : a journal of the British Diabetic Association. 2018; 142:612. 
15. Tancredi M, Rosengren A, Svensson A-M, et al. Excess Mortality among Persons with Type 2 Diabetes. The New England journal of medicine. 2015; 373(18):1720-32. [PubMed: 26510021]

16. Eeg-Olofsson K, Cederholm J, Nilsson PM, et al. Glycemic control and cardiovascular disease in 7,454 patients with type 1 diabetes: an observational study from the Swedish National Diabetes Register (NDR). Diabetes Care. 2010; 33(7):1640-6. [PubMed: 20424222]

17. Rawshani A, Landin-Olsson M, Svensson A-M, et al. The incidence of diabetes among 0-34 year olds in Sweden: new data and better methods. Diabetologia. 2014; 57(7):1375-81. [PubMed: 24710965]

18. Lind M, Bounias I, Olsson M, Gudbjörnsdottir S, Svensson A-M, Rosengren A. Glycaemic control and incidence of heart failure in 20,985 patients with type 1 diabetes: an observational study. Lancet. 2011; 378(9786):140-6. [PubMed: 21705065]

19. Ludvigsson JF, Andersson E, Ekbom A, et al. External review and validation of the Swedish national inpatient register. BMC Public Health. 2011; 11(1):450. [PubMed: 21658213]

20. The incidence of diabetes among 0-34 year olds in Sweden: new data and better methods. 2014; 57(7):1375-81.

21. Nordestgaard BG, Chapman MJ, Humphries SE, et al. Familial hypercholesterolaemia is underdiagnosed and undertreated in the general population: guidance for clinicians to prevent coronary heart disease: consensus statement of the European Atherosclerosis Society. 2013:347890a.

22. Prospective Studies Collaboration and Asia Pacific Cohort Studies Collaboration. Sex-specific relevance of diabetes to occlusive vascular and other mortality: a collaborative meta-analysis of individual data from 980793 adults from 68 prospective studies. Lancet Diabetes Endocrinol. 2018

23. Ference BA, Ginsberg HN, Graham I, et al. Low-density lipoproteins cause atherosclerotic cardiovascular disease. 1. Evidence from genetic, epidemiologic, and clinical studies. A consensus statement from the European Atherosclerosis Society Consensus Panel. Eur Heart J. 2017; 38(32): 2459-72. [PubMed: 28444290]

24. Paneni F, Beckman JA, Creager MA, Cosentino F. Diabetes and vascular disease: pathophysiology, clinical consequences, and medical therapy: part I. Eur Heart J. 2013; 34(31):2436-43. [PubMed: 23641007]

25. Leete P, Willcox A, Krogvold L, et al. Differential Insulitic Profiles Determine the Extent of $\beta$-Cell Destruction and the Age at Onset of Type 1 Diabetes. Diabetes. 2016; 65(5):1362-9. [PubMed: 26858360]

26. Arif S, Leete P, Nguyen V, et al. Blood and islet phenotypes indicate immunological heterogeneity in type 1 diabetes. Diabetes. 2014; 63(11):3835-45. [PubMed: 24939426]

27. Singh TP, Groehn H, Kazmers A. Vascular function and carotid intimal-medial thickness in children with insulin-dependent diabetes mellitus. J Am Coll Cardiol. 2003; 41(4):661-5. [PubMed: 12598080]

28. Järvisalo MJ, Raitakari M, Toikka JO, et al. Endothelial dysfunction and increased arterial intimamedia thickness in children with type 1 diabetes. Circulation. 2004; 109(14):1750-5. [PubMed: 15023875]

29. Costacou T, Lopes-Virella MF, Zgibor JC, et al. Markers of endothelial dysfunction in the prediction of coronary artery disease in type 1 diabetes. The Pittsburgh Epidemiology of Diabetes Complications Study. J Diabetes Complicat. 2005; 19(4):183-93. [PubMed: 15993351]

30. Vinik AI, Ziegler D. Diabetic cardiovascular autonomic neuropathy. Circulation. 2007; 115(3): 387-97. [PubMed: 17242296]

31. Margeirsdottir HD, Stensaeth KH, Larsen JR, Brunborg C, Dahl-Jørgensen K. Early signs of atherosclerosis in diabetic children on intensive insulin treatment: a population-based study. Diabetes Care. 2010; 33(9):2043-8. [PubMed: 20530748]

32. Cholesterol Treatment Trialists' (CTT) Collaborators. Kearney PM, Blackwell L, et al. Efficacy of cholesterol-lowering therapy in 18,686 people with diabetes in 14 randomised trials of statins: a meta-analysis. Lancet. 2008; 371(9607):117-25. [PubMed: 18191683]

33. Haller MJ, Stein JM, Shuster JJ, et al. Pediatric Atorvastatin in Diabetes Trial (PADIT): a pilot study to determine the effect of atorvastatin on arterial stiffness and endothelial function in 
children with type 1 diabetes mellitus. J Pediatr Endocrinol Metab. 2009; 22(1):65-8. [PubMed: 19344076]

34. de Ferranti SD, de Boer IH, Fonseca V, et al. Type 1 Diabetes Mellitus and Cardiovascular Disease: A Scientific Statement From the American Heart Association and American Diabetes Association. Circulation. 2014; 130(13):1110-30. [PubMed: 25114208]

35. Hero C, Rawshani A, Svensson A-M, et al. Association Between Use of Lipid-Lowering Therapy and Cardiovascular Diseases and Death in Individuals With Type 1 Diabetes. Diabetes Care. 2016

36. Marcovecchio ML, Chiesa ST, Bond S, et al. ACE Inhibitors and Statins in Adolescents with Type 1 Diabetes. The New England journal of medicine. 2017; 377(18):1733-45. [PubMed: 29091568]

37. Livingstone SJ, Looker HC, Hothersall EJ, et al. Risk of cardiovascular disease and total mortality in adults with type 1 diabetes: Scottish registry linkage study. PLoS Med. 2012; 9(10):e1001321. [PubMed: 23055834]

38. JBS3 Board. Joint British Societies' consensus recommendations for the prevention of cardiovascular disease (JBS3). Heart. 2014; 100(Suppl 2):ii1-ii67. [PubMed: 24667225]

39. Ford I, Murray H, Packard CJ, et al. Long-term follow-up of the West of Scotland Coronary Prevention Study. The New England journal of medicine. 2007; 357(15):1477-86. [PubMed: 17928595] 


\section{Evidence before this study}

People with type 1 diabetes are at 2- to 5-fold increased risk of death and 3- to 7-fold increased risk of coronary heart disease. Several risk factors, notably glycemic control, affect survival in type 1 diabetes. The importance of age at disease onset, however, remains weakly studied. Guidelines do not articulate any specific recommendations in relation to age at disease onset, only duration. We did a systematic search in PubMed for articles published between Jan 1, 1960, and April 15, 2018. Our search terms included "type 1 diabetes", "age at diagnosis", "age at disease onset", "childhood onset", "late onset", "debut age", "mortality", "cardiovascular disease", "coronary artery disease", "myocardial infarction". We searched articles by title and abstract to identify relevant studies. Studies were also sought within reference lists of eligible studies. We considered studies that evaluated association between age at onset/diagnosis of type 1 diabetes and cardiovascular disease and survival. Studies using diabetes free controls as comparator were of primary interest, as such studies addresses the excess risk conferred by diabetes.

\section{Added value of this study}

By studying 27,195 individuals with type 1 diabetes and 135,178 matched controls, we demonstrate a ubiquitous inverse association between age at diagnosis and risk of mortality and cardiovascular disease, independent of diabetes duration. Patients with type 1 diabetes with disease onset before 10 years of age experienced a 30-fold increased risk of CHD and AMI. Women with onset of type 1 diabetes before 10 years of age displayed a 60- and 90-fold increased risk of CHD and AMI, respectively. The difference in risk levels between those with onset at age 0-10 years and 25-30 years was up to 5-fold (AMI and CHD). Although absolute risks were low in this young cohort, developing T1D before 10 years of age resulted in a loss of 17.7 and 14.2 life years for women and men, respectively, whereas years of life lost were around 9-10 years with later age at diagnosis.

\section{Implications of all the available evidence}

Age at disease onset appears an important determinant of survival and, in particular of, cardiovascular disease in type 1 diabetes. These findings suggest that more patients with earlier onset type 1 diabetes be offered cardioprotective medications (statins, BP medications) sooner than currently practiced. A greater effort towards improved glycaemia control in such individual would also be beneficial. 


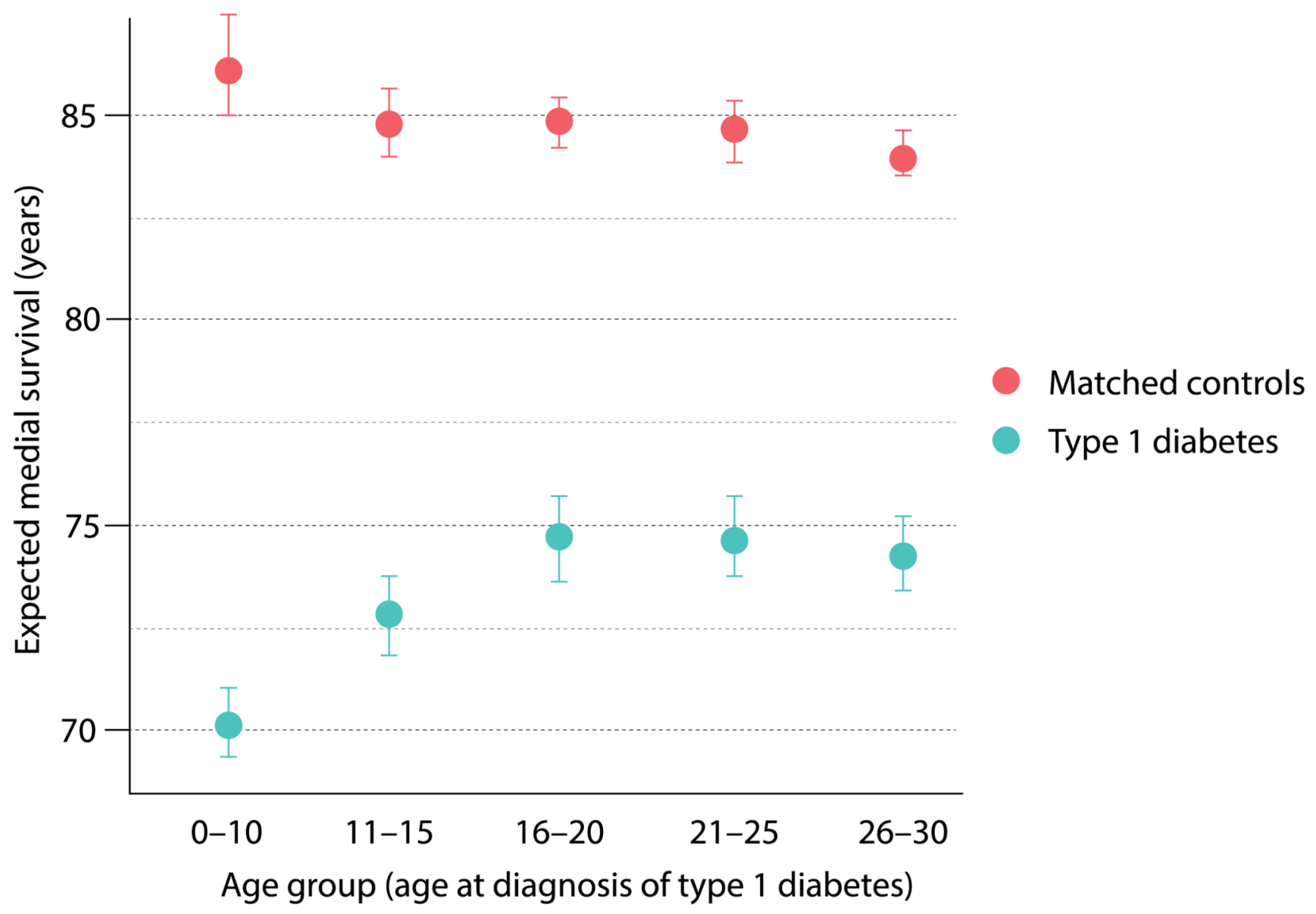

Figure 1. Life years lost in relation to age at onset of type 1 diabetes

Loss of life year was estimated using separate Cox regressions fitted to persons with type 1 diabetes and their control persons within each age group. Conditional median survival was estimated from the upper limit of each age interval. Life years lost due to diabetes is calculated as the difference (between people with type 1 diabetes and controls) in the expected median survival. 

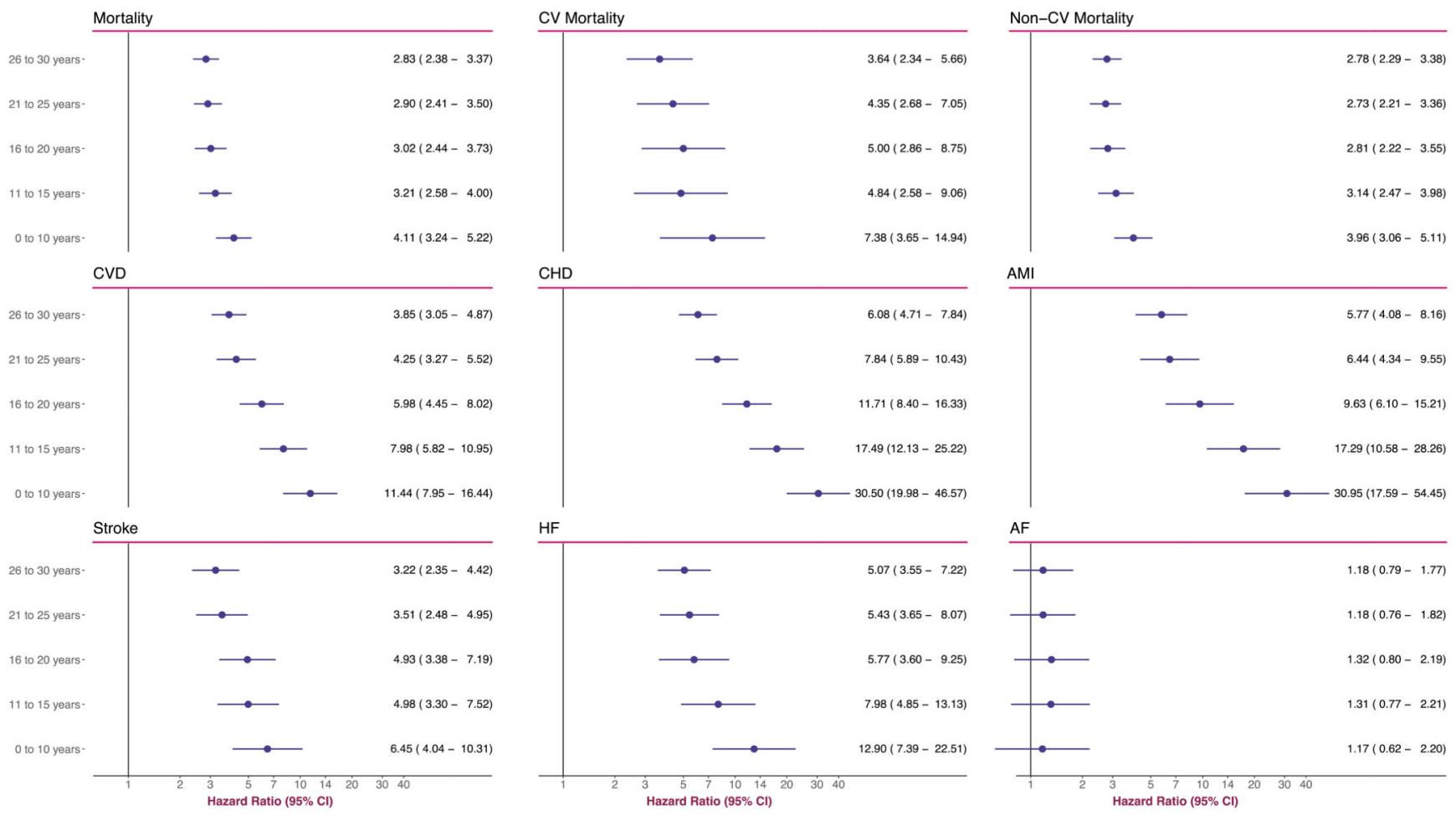

Figure 2. Adjusted Hazard Ratios for All Outcomes, According to Age at T1D Diagnosis The analyses were based on Cox regression and adjusted was made for preexisting comorbidities, calendar year, income, country of birth, marital status, educational attainment, duration of diabetes. Matched controls serve as reference group for all models. 

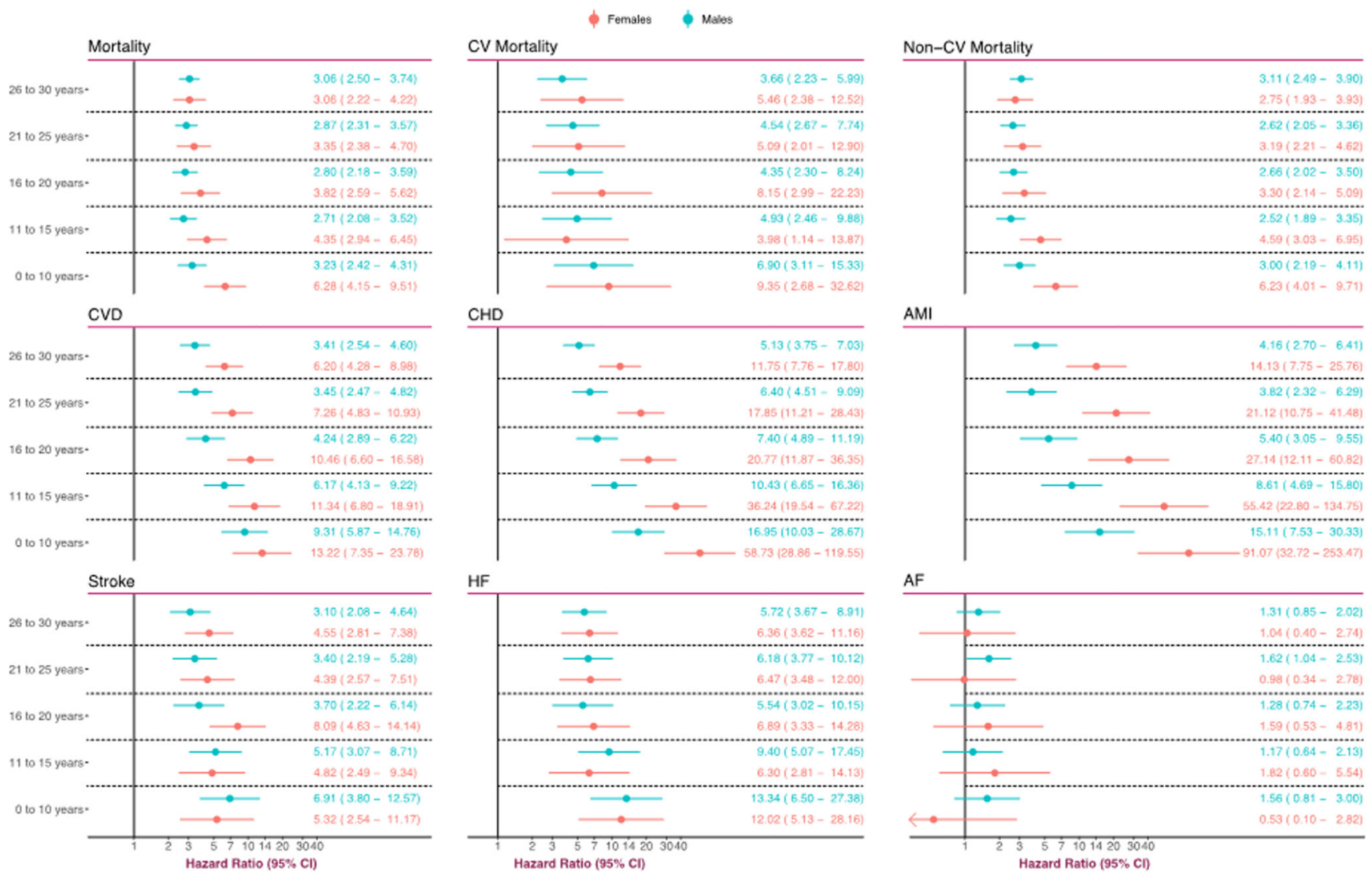

Figure 3. Adjusted Hazard Ratios for All Outcomes, According to Age at T1D Diagnosis and Stratified by Sex

The analyses were based on Cox regression and adjusted was made for preexisting comorbidities, calendar year, income, country of birth, marital status, educational attainment, duration of diabetes. Matched controls serve as reference group for all models. 

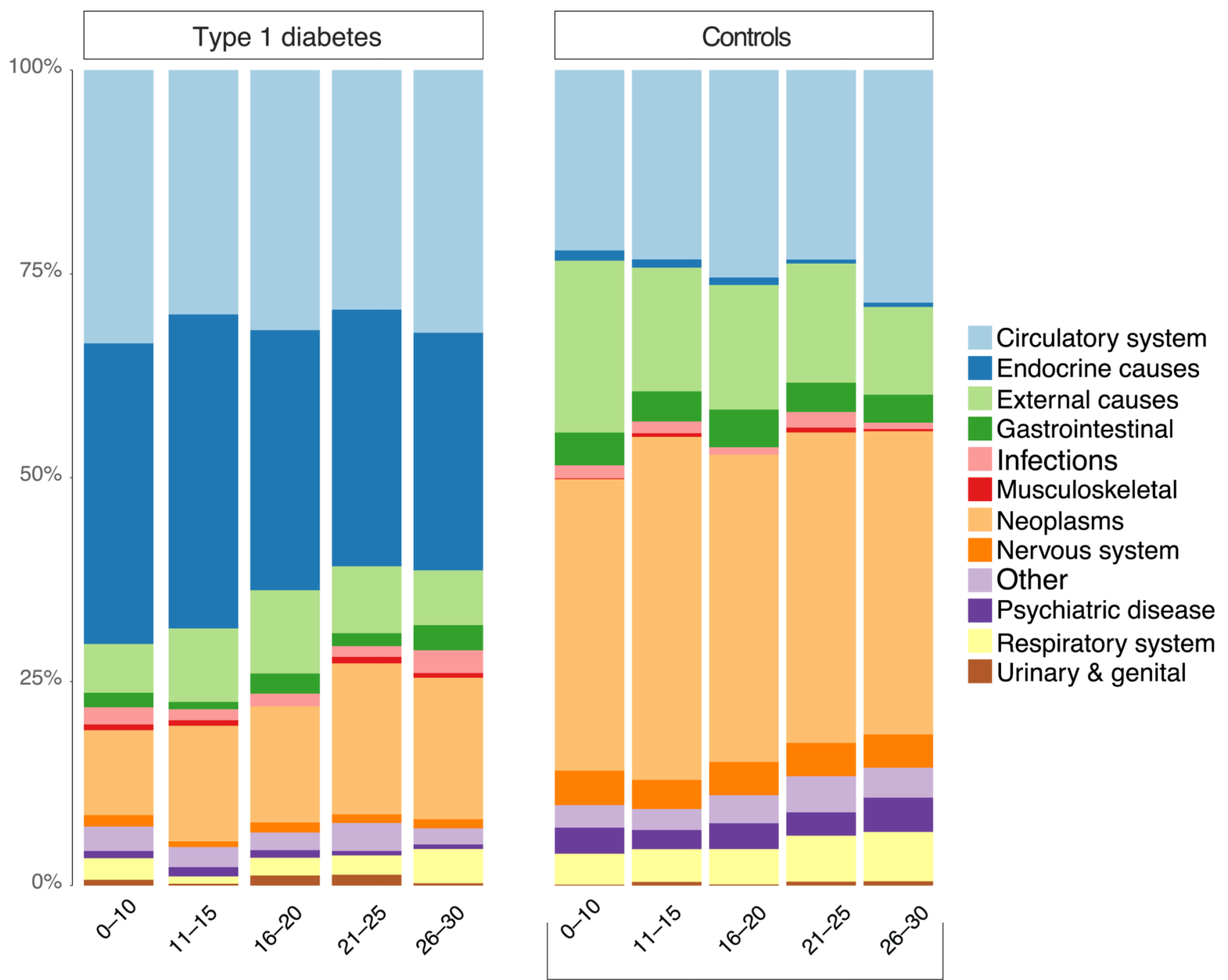

Age-group allocation according to matching

Figure 4. Causes of Death According to Age at Diagnosis of type 1 diabetes

Causes of death according death certificates in the Swedish Cause of Death Registry. Causes of death in the registry are classified according to the International Classification of Diseases (ICD), 10th Revision. Only the primary cause of death was assessed. Hence, cardiovascular disease may be a significant contributor to deaths classified as e.g "Endocrine causes". 
Table 1

Descriptive statistics for persons with diabetes and matched controls

\begin{tabular}{|c|c|c|c|c|c|c|c|}
\hline & \multirow[t]{3}{*}{ Controls } & \multicolumn{6}{|c|}{ Individuals with type 1 diabetes } \\
\hline & & \multirow[t]{2}{*}{ All } & \multicolumn{5}{|c|}{ According to age at diagnosis } \\
\hline & & & 0-10 years & 11-15 years & $16-20$ years & 21-25 years & 26-30 years \\
\hline $\mathrm{n}$ & 135178 & 27195 & 7409 & 6538 & 4619 & 4616 & 4013 \\
\hline Male $(\%)$ & $\begin{array}{l}75417 \\
(55.8)\end{array}$ & $\begin{array}{l}15165 \\
(55.8)\end{array}$ & $3741(50.5)$ & $3652(55.9)$ & $2760(59.8)$ & $2682(58.1)$ & $2330(58.1)$ \\
\hline Age - mean $(\mathrm{SD})$ & $28.84(9.34)$ & $\begin{array}{l}28.86 \\
(9.36)\end{array}$ & $23.75(5.39)$ & $26.05(7.33)$ & $28.36(9.03)$ & $33.25(9.35)$ & $38.40(9.18)$ \\
\hline \multicolumn{8}{|l|}{ Education (\%) } \\
\hline College level & $\begin{array}{l}71900 \\
(54.3)\end{array}$ & $\begin{array}{l}15009 \\
(56.0)\end{array}$ & $4252(58.3)$ & $3694(57.4)$ & $2599(57.1)$ & $2432(53.3)$ & $2032(51.2)$ \\
\hline Elementary school & $\begin{array}{l}25426 \\
(19.2)\end{array}$ & $\begin{array}{l}5424 \\
(20.2)\end{array}$ & $1642(22.5)$ & $1337(20.8)$ & $930(20.4)$ & $770(16.9)$ & $745(18.8)$ \\
\hline Upper secondary school & $\begin{array}{l}35130 \\
(26.5)\end{array}$ & $\begin{array}{l}6381 \\
(23.8)\end{array}$ & 1405 (19.2) & $1410(21.9)$ & $1020(22.4)$ & $1358(29.8)$ & $1188(30.0)$ \\
\hline \multicolumn{8}{|l|}{ Marital status (\%) } \\
\hline Married & $\begin{array}{l}29673 \\
(22.0)\end{array}$ & $\begin{array}{l}5606 \\
(20.6)\end{array}$ & $682(9.2)$ & $954(14.6)$ & $888(19.2)$ & $1445(31.3)$ & $1637(40.8)$ \\
\hline Divorced & $5996(4.4)$ & $1263(4.6)$ & $88(1.2)$ & $204(3.1)$ & $218(4.7)$ & $337(7.3)$ & $416(10.4)$ \\
\hline Single & $\begin{array}{l}99264 \\
(73.4)\end{array}$ & $\begin{array}{l}20284 \\
(74.6)\end{array}$ & $6637(89.6)$ & $5369(82.1)$ & $3510(76.0)$ & $2820(61.1)$ & $1948(48.5)$ \\
\hline Widowed & $220(0.2)$ & $41(0.2)$ & $2(0.0)$ & $10(0.2)$ & $3(0.1)$ & $14(0.3)$ & $12(0.3)$ \\
\hline \multicolumn{8}{|l|}{ Region of birth (\%) } \\
\hline Other European Union & $2402(1.9)$ & $264(1.0)$ & $52(0.7)$ & $55(0.9)$ & $39(0.9)$ & $68(1.5)$ & $50(1.3)$ \\
\hline Nordic & $2473(1.9)$ & $490(1.9)$ & $91(1.2)$ & $90(1.4)$ & $85(1.9)$ & $114(2.6)$ & $110(2.9)$ \\
\hline Non-European & $5924(4.6)$ & $543(2.1)$ & $83(1.1)$ & $92(1.4)$ & $77(1.7)$ & $124(2.8)$ & $167(4.4)$ \\
\hline Sweden & $\begin{array}{l}117188 \\
(91.6)\end{array}$ & $\begin{array}{l}25132 \\
(95.1)\end{array}$ & 7103 (96.9) & $6215(96.3)$ & $4271(95.5)$ & $4096(93.0)$ & 3447 (91.3) \\
\hline Income $^{1}$ - mean $(\mathrm{SD})$ & $\begin{array}{l}1308.20 \\
(1257.71)\end{array}$ & $\begin{array}{l}1261.64 \\
(992.00)\end{array}$ & $\begin{array}{l}1002.10 \\
(677.80)\end{array}$ & $\begin{array}{l}1141.09 \\
(869.05)\end{array}$ & $\begin{array}{l}1229.59 \\
(919.07)\end{array}$ & $\begin{array}{l}1509.13 \\
(1084.74)\end{array}$ & $\begin{array}{l}1689.42 \\
(1364.79)\end{array}$ \\
\hline \multicolumn{8}{|l|}{$\begin{array}{l}\text { Preexisting conditions - } \\
\mathrm{n}(\%)\end{array}$} \\
\hline CVD & $209(0.2)$ & $240(0.9)$ & $27(0.4)$ & $34(0.5)$ & $37(0.8)$ & $64(1.4)$ & $78(1.9)$ \\
\hline CHD & $160(0.1)$ & $252(0.9)$ & $20(0.3)$ & $32(0.5)$ & $36(0.8)$ & $74(1.6)$ & $90(2.2)$ \\
\hline AMI & $83(0.1)$ & $142(0.5)$ & $11(0.1)$ & $17(0.3)$ & $21(0.5)$ & $44(1.0)$ & $49(1.2)$ \\
\hline Stroke & $127(0.1)$ & $110(0.4)$ & $17(0.2)$ & $18(0.3)$ & $17(0.4)$ & $24(0.5)$ & $34(0.8)$ \\
\hline Renal disease & $27(0.0)$ & $110(0.4)$ & $20(0.3)$ & $33(0.5)$ & $25(0.5)$ & $19(0.4)$ & $13(0.3)$ \\
\hline Heart failure & $47(0.0)$ & $92(0.3)$ & $9(0.1)$ & $8(0.1)$ & $14(0.3)$ & $23(0.5)$ & $38(0.9)$ \\
\hline Atrial fibrillation & $128(0.1)$ & $36(0.1)$ & $2(0.0)$ & $4(0.1)$ & $4(0.1)$ & $13(0.3)$ & $13(0.3)$ \\
\hline Amputation & $8(0.0)$ & $36(0.1)$ & $3(0.0)$ & $5(0.1)$ & $6(0.1)$ & $7(0.2)$ & $15(0.4)$ \\
\hline Hbalc - mean (SD) & & & $\begin{array}{l}69.75 \\
(15.67)\end{array}$ & $\begin{array}{l}67.61 \\
(15.91)\end{array}$ & $\begin{array}{l}63.44 \\
(17.04)\end{array}$ & $63.09(17.52)$ & $63.63(17.53)$ \\
\hline
\end{tabular}




\begin{tabular}{|c|c|c|c|c|c|c|c|}
\hline & \multirow[t]{3}{*}{ Controls } & \multicolumn{6}{|c|}{ Individuals with type 1 diabetes } \\
\hline & & \multirow[t]{2}{*}{ All } & \multicolumn{5}{|c|}{ According to age at diagnosis } \\
\hline & & & $0-10$ years & $11-15$ years & 16-20 years & 21-25 years & 26-30 years \\
\hline $\begin{array}{l}\text { Systolic blood pressure - } \\
\text { mean (SD) }\end{array}$ & & & $\begin{array}{l}120.19 \\
(12.49)\end{array}$ & $\begin{array}{l}121.46 \\
(13.20)\end{array}$ & $\begin{array}{l}122.22 \\
(14.12)\end{array}$ & $\begin{array}{l}124.03 \\
(15.16)\end{array}$ & $\begin{array}{l}126.28 \\
(16.24)\end{array}$ \\
\hline $\mathrm{BMI}-$ mean $(\mathrm{SD})$ & & & $24.56(3.58)$ & $24.63(3.76)$ & $24.67(4.11)$ & $25.40(4.57)$ & $26.15(4.99)$ \\
\hline $\begin{array}{l}\text { LDL cholesterol - mean } \\
\text { (SD) }\end{array}$ & & & $2.55(0.80)$ & $2.59(0.81)$ & $2.58(0.80)$ & $2.66(0.81)$ & $2.83(0.91)$ \\
\hline $\begin{array}{l}\text { HDL cholesterol - mean } \\
\text { (SD) }\end{array}$ & & & $1.48(0.40)$ & $1.49(0.41)$ & $1.44(0.42)$ & $1.46(0.46)$ & $1.44(0.43)$ \\
\hline $\begin{array}{l}\text { Estimated } \mathrm{GFR}^{2} \text { - mean } \\
\text { (SD) }\end{array}$ & & & $\begin{array}{l}108.44 \\
(27.82)\end{array}$ & $\begin{array}{l}109.24 \\
(33.31)\end{array}$ & $\begin{array}{l}106.73 \\
(28.42)\end{array}$ & $\begin{array}{l}101.65 \\
(28.48)\end{array}$ & $98.02(28.33)$ \\
\hline $\begin{array}{l}\text { Lipid lowering medication } \\
-\mathrm{n}(\%)\end{array}$ & & & $0.03(0.16)$ & $0.03(0.18)$ & $0.05(0.22)$ & $0.09(0.28)$ & $0.12(0.32)$ \\
\hline Antihypertensives $-\mathrm{n}(\%)$ & & & $0.10(0.29)$ & $0.11(0.31)$ & $0.10(0.30)$ & $0.13(0.33)$ & $0.17(0.38)$ \\
\hline Smokers $-\mathrm{n}(\%)$ & & & $868(12.8)$ & $808(13.5)$ & $521(12.3)$ & $650(15.4)$ & $648(17.8)$ \\
\hline $\begin{array}{l}\text { No physical activity }{ }^{3}-\mathrm{n} \\
(\%)\end{array}$ & & & $215(7.9)$ & $170(7.9)$ & $106(7.8)$ & $104(9.2)$ & $83(8.4)$ \\
\hline $\begin{array}{l}\text { Daily physical }{ }^{3} \text { activity - } \\
\mathrm{n}(\%)\end{array}$ & & & $550(20.2)$ & $438(20.4)$ & $318(23.3)$ & $248(21.9)$ & $235(23.8)$ \\
\hline $\begin{array}{l}\text { Microalbuminuria }{ }^{4}-\mathrm{n} \\
(\%)\end{array}$ & & & $439(7.6)$ & $352(6.8)$ & $205(5.6)$ & $242(6.7)$ & $241(7.7)$ \\
\hline $\begin{array}{l}\text { Macroalbuminuria }{ }^{4}-\mathrm{n} \\
(\%)\end{array}$ & & & $258(4.5)$ & $256(5.0)$ & $142(3.9)$ & $180(5.0)$ & $157(5.0)$ \\
\hline
\end{tabular}

Abbreviations: $\mathrm{CVD}=$ cardiovascular disease CHD = coronary heart disease $; \mathrm{AMI}=$ acute myocardial infarction; $\mathrm{BMI}=$ body mass index; $\mathrm{LDL}$ = low density lipoprotein; HDL = high density lipoprotein; GFR = glomerular filtration rate.

1 Income in 100 SEK / years. $100 \mathrm{SEK} \approx £ 8.54 \approx \$ 11.45$.

2 The estimated glomerular filtration rate (eGFR) was calculated with the Modification of Diet in Renal Disease equation (Levey et al. Ann Intern Med. 1999)

3 Physical activity at least 30 minutes per day.

4 Microalbuminuria was defined by two positive results on three urine samples obtained within 1 year, with positivity defined by an albumin: creatinine ratio of 3 to $30 \mathrm{mg}$ per millimole (approximately 30 to $300 \mathrm{mg}$ per gram) or a urinary albumin clearance of 20 to $200 \mu \mathrm{g}$ per minute [20 to $300 \mathrm{mg}$ per liter]). Macroalbuminuria was defined by an albumin:creatinine ratio of more than $30 \mathrm{mg}$ per millimole (approximately $>300 \mathrm{mg}$ per gram) or a urinary albumin clearance of more than $200 \mu \mathrm{g}$ per minute (>300 $\mathrm{mg}$ per liter). 